\title{
Low energy universality and scaling of Van der Waals forces
}

\author{
A. Calle Cordón ${ }^{1, *}$ and E. Ruiz Arriola ${ }^{1, \dagger}$ \\ ${ }^{1}$ Departamento de Física Atómica, Molecular y Nuclear, \\ Universidad de Granada, E-18071 Granada, Spain.
}

(Dated: October 29, 2018)

\begin{abstract}
At long distances interactions between neutral ground state atoms can be described by the Van der Waals potential $V(r)=-\sum_{n=6}^{\infty} C_{n} / r^{n}$. In the ultra-cold regime atom-atom scattering is dominated by s-waves phase shifts given by an effective range expansion $p \cot \delta_{0}(p)=-1 / \alpha_{0}+r_{0} p^{2} / 2+\ldots$ in terms of the scattering length $\alpha_{0}$ and the effective range $r_{0}$. We show that while for these potentials the scattering length cannot be predicted, the effective range is given by the universal low energy theorem $r_{0}=A+B / \alpha_{0}+C / \alpha_{0}^{2}$ where $A, B$ and $C$ depend on the dispersion coefficients $C_{n}$ and the reduced di-atom mass. We confront this formula to about a hundred determinations of $r_{0}$ and $\alpha_{0}$ and show why the result is dominated by the leading dispersion coefficient $C_{6}$. Universality and scaling extends much beyond naive dimensional analysis estimates.
\end{abstract}

PACS numbers: 34.10.+x; 34.50.Cx; 33.15.-e; 03.75.Nt

Keywords: Atom-atom scattering, Van der Waals forces, Scattering length, Effective range, Renormalization

Van der Waals (VdW) forces appear ubiquitously in many contexts of atomic, molecular, nuclear and particle physics. They account for long range dipole fluctuations between charge neutral atomic and molecular systems [1] with implications on the production of Bose-Einstein condensates of ultra-cold atoms and molecules [2]. The intermediate range nucleon-nucleon interaction due to two pion exchange also exhibits this $\mathrm{VdW}$ behaviour based on chiral symmetry [3] providing a justification for the liquid drop model of nuclei [4]. The short distance gluon exchange interaction between (colour neutral) hadrons also display this kind of interaction [5, 6]. Van der Waals forces, however, diverge when naively extrapolated to short distance scales [7, 8]. The study of such problems in a variety of situations will certainly shed light on the usefulness of renormalization ideas within the specific context of quantum mechanics (see e.g. Ref. [9]).

Fundamental work for neutral atoms was initiated in Refs. 10, 11, 12] (see also [13]), within a quantum-defect theoretical viewpoint. In this letter we systematically show that these simplified approaches work and analyze why they succeed. VdW forces are extremely simple in this case and are described by the potential

$$
V(r)=-\sum_{n=6}^{\infty} \frac{C_{n}}{r^{n}}
$$

where $C_{n}$ are the $\mathrm{VdW}$ coefficients which are computed $a b$ initio from intensive electronic orbital atomic structure calculations (see e.g. Ref. 14] for a compilation). Usually, only the terms with $n=6,8,10$ are retained although the series is expected to diverge asymptotically, $C_{n} \sim n$ ! 15]. The impressive calculation in Hydrogen up to $C_{32}$ [16] exhibits the behaviour $C_{n} \sim(1 / 2)^{n} n$ ! at relatively low $n$-values. The potential (1) holds for distances much larger than the ionization length $l_{I}=\hbar / \sqrt{2 m_{e} I}$ ( $I$ is the ionization potential) which usually is a few a.u. In the Born-Oppenheimer approximation the quantum mechanical problem consists of solving the Schrödinger equation for the two atoms apart a distance $r$,

$$
-u_{k}^{\prime \prime}+U(r) u_{k}+\frac{l(l+1)}{r^{2}} u_{k}=k^{2} u_{k},
$$

where $U(r)=2 \mu V(r) / \hbar^{2}$ is the reduced potential, $\mu=$ $m_{1} m_{2} /\left(m_{1}+m_{2}\right)$ the reduced di-atom mass, $k=p / \hbar=$ $2 \pi / \lambda$ the wavenumber, and $u_{k}(r)$ the reduced wave function. For our purposes, it is convenient to write the reduced potential in VdW units,

$$
U(r)=-\frac{R_{6}^{4}}{r^{6}}\left[1+g_{1} \frac{R_{6}^{2}}{r^{2}}+g_{2} \frac{R_{6}^{4}}{r^{4}}+\ldots\right],
$$

where $R_{6}=\left(2 \mu C_{6} / \hbar^{2}\right)^{\frac{1}{4}}$ is the $\mathrm{VdW}$ length scale and $g_{n}=\left(2 \mu C_{6+2 n} / \hbar^{2}\right) R_{6}^{-4-2 n}$ for $n \geq 1$. In Table 【 we display numerical values for several di-atomic systems which are extremely small in the VdW units $g_{1} \sim 10^{-2}$ and $g_{2} \sim 10^{-4}$ with the exception of $\mathrm{H}$ and He. Thus, we may anticipate a dominance of the $C_{6}$ term in the calculations at low energies. This will be systematically quantified below.

Using the superposition principle for positive energy scattering s-waves we decompose the general solution as

$$
u_{k}(r)=u_{k, c}(r)+k \cot \delta_{0}(k) u_{k, s}(r),
$$

with $u_{k, c}(r) \rightarrow \cos (k r)$ and $u_{k, s}(r) \rightarrow \sin (k r) / k$ for $r \rightarrow \infty$ and $\delta_{0}(k)$ the scattering phase shift for the $l=0$ angular momentum state. The potential given by Eq. (1) is both long range and singular at short distances. At short distances, the De Broglie wavelength is slowly varying and hence a WKB approximation holds [7, 8], yielding for $r \rightarrow 0$

$$
u_{k}(r) \rightarrow C\left(\frac{r}{R_{n}}\right)^{\frac{n}{4}} \sin \left[\frac{2}{n-2}\left(\frac{R_{n}}{r}\right)^{\frac{n}{2}-1}+\varphi_{k}\right]
$$


TABLE I: Van der Waals length $R_{6}=\left(2 \mu C_{6} / \hbar^{2}\right)^{\frac{1}{4}}$, and the coefficients $g_{1}$ and $g_{2}$ defined by the reduced dimensionless potential $2 \mu V(r) R_{6}^{2} / \hbar^{2} \equiv-x^{-6}\left[1+g_{1} x^{-2}+g_{2} x^{-4}+\ldots\right]$ with $x=r / R_{6}$. We use results from Refs. [17, 18, 19, 20, 21, 22]

\begin{tabular}{|c|c|c|c|}
\hline \hline Atoms & $R_{6}$ (a.u.) & $g_{1}\left(10^{-2}\right)$ & $g_{2}\left(10^{-4}\right)$ \\
\hline $\mathrm{Li}-\mathrm{Li}$ & 64.9214 & 1.42458 & 2.97874 \\
\hline $\mathrm{Na}-\mathrm{Na}$ & 89.8620 & 0.92320 & 1.11369 \\
\hline $\mathrm{K}-\mathrm{K}$ & 128.9846 & 0.64780 & 0.49784 \\
\hline $\mathrm{Rb}-\mathrm{Rb}$ & 164.1528 & 0.45647 & 0.23370 \\
\hline $\mathrm{Cs}-\mathrm{Cs}$ & 201.8432 & 0.36544 & 0.13983 \\
\hline $\mathrm{Fr}-\mathrm{Fr}$ & 215.0006 & 0.27362 & 0.09526 \\
\hline $\mathrm{Li}-\mathrm{Na}$ & 73.2251 & 1.25605 & 2.17183 \\
\hline $\mathrm{Li}-\mathrm{K}$ & 84.2285 & 1.18374 & 1.79689 \\
\hline $\mathrm{Li}-\mathrm{Rb}$ & 88.0587 & 1.18572 & 1.70555 \\
\hline $\mathrm{Li}-\mathrm{Cs}$ & 92.8950 & 1.21364 & 1.68241 \\
\hline $\mathrm{Na}-\mathrm{K}$ & 106.5708 & 0.80600 & 0.80155 \\
\hline $\mathrm{Na}-\mathrm{Rb}$ & 115.3377 & 0.74528 & 0.65923 \\
\hline $\mathrm{Na}-\mathrm{Cs}$ & 123.2277 & 0.73874 & 0.61148 \\
\hline $\mathrm{K}-\mathrm{Rb}$ & 142.8292 & 0.56543 & 0.37106 \\
\hline $\mathrm{K}-\mathrm{Cs}$ & 154.2909 & 0.53903 & 0.32152 \\
\hline $\mathrm{Rb}-\mathrm{Cs}$ & 180.8480 & 0.41520 & 0.18654 \\
\hline $\mathrm{Be}-\mathrm{Be}$ & 43.3013 & 2.54953 & 6.70303 \\
\hline $\mathrm{Mg}-\mathrm{Mg}$ & 72.3589 & 1.26414 & 1.60399 \\
\hline $\mathrm{Ca}-\mathrm{Ca}$ & 111.4907 & 0.84584 & 0.65057 \\
\hline $\mathrm{Sr}-\mathrm{Sr}$ & 148.9023 & 0.55117 & 0.27632 \\
\hline $\mathrm{Ba}-\mathrm{Ba}$ & 189.4340 & 0.41692 & 0.15260 \\
\hline $\mathrm{Cr}-\mathrm{Cr}$ & 91.2731 & 1.22821 & - \\
\hline \hline $\mathrm{H}-\mathrm{H}$ & 10.4532 & 17.51760 & 423.45426 \\
\hline $\mathrm{He}-\mathrm{He}$ & 10.1610 & 9.35937 & 117.94642 \\
\hline \hline & & & \\
\hline
\end{tabular}

where $R_{n}=\left(2 \mu C_{n} / \hbar^{2}\right)^{1 /(n-2)}$ corresponds to the highest $\mathrm{VdW}$ scale considered in Eq. (1). The phase $\varphi_{k}$ is in principle arbitrary. On the other hand, at low energies one has the effective range expansion [23]

$$
k \cot \delta_{0}(k)=-\frac{1}{\alpha_{0}}+\frac{1}{2} r_{0} k^{2}+v_{2} k^{4} \log \left(k^{2}\right)+\ldots
$$

where $\alpha_{0}$ is the scattering length, and $r_{0}$ is the effective range which can be calculated from

$$
r_{0}=2 \int_{0}^{\infty} d r\left[\left(1-\frac{r}{\alpha_{0}}\right)^{2}-u_{0}(r)^{2}\right]
$$

Here, the zero energy solution becomes, from Eq. (4),

$$
u_{0}(r)=u_{0, c}(r)-u_{0, s}(r) / \alpha_{0}
$$

where $u_{0, c}(r) \rightarrow 1$ and $u_{0, s}(r) \rightarrow r$ for $r \rightarrow \infty$, yielding

$$
r_{0}=A+\frac{B}{\alpha_{0}}+\frac{C}{\alpha_{0}^{2}}
$$

with $A, B$ and $C$ given by

$$
\begin{aligned}
& A=2 \int_{0}^{\infty} d r\left(1-u_{0, c}^{2}\right) \\
& B=-4 \int_{0}^{\infty} d r\left(r-u_{0, c} u_{0, s}\right) \\
& C=2 \int_{0}^{\infty} d r\left(r^{2}-u_{0, s}^{2}\right)
\end{aligned}
$$

Then, combining the zero and finite energy wave functions we get for any $r_{c}>0$

$$
u_{k}^{\prime}(r) u_{0}(r)-\left.u_{0}^{\prime}(r) u_{k}(r)\right|_{r_{c}} ^{\infty}=k^{2} \int_{r_{c}}^{\infty} u_{k}(r) u_{0}(r) d r
$$

where $r_{c}$ plays the role of a short distance cut-off which is innocuous provided $l_{I} \ll r_{c} \ll R_{n}$. Using Eq. (4), Eq. (5) and Eq. (8) we then get for $r_{c} \rightarrow 0$

$$
\begin{aligned}
\frac{1}{R_{n}} \sin \left(\varphi_{k}-\varphi_{0}\right) & =k^{2} \int_{0}^{\infty} d r\left[u_{0, c}(r)-\frac{1}{\alpha_{0}} u_{0, s}(r)\right] \\
& \times\left[u_{k, c}(r)+k \cot \delta_{0}(k) u_{k, s}(r)\right] .(14)
\end{aligned}
$$

Orthogonality between $u_{k}$ and $u_{0}$ requires $\varphi_{k}=\varphi_{0}$ in which case, expanding the integrand we get the structure

$$
k \cot \delta_{0}(k)=\frac{\alpha_{0} \mathcal{A}(k)+\mathcal{B}(k)}{\alpha_{0} \mathcal{C}(k)+\mathcal{D}(k)},
$$

where $\mathcal{A}, \mathcal{B}, \mathcal{C}$ and $\mathcal{D}$ are suitable functions of $k$. The interesting feature of Eqs. (9) and (15) is that the dependence on the scattering length $\alpha_{0}$ and the potential is explicitly disentangled. This is a universal form of a low energy theorem, which applies to any potential regular or singular at the origin which falls off faster than $1 / r^{5}$ at large distances. We can visualize Eq. (9) as a long distance $(\mathrm{VdW})$ correlation between $r_{0}$ and $\alpha_{0}$. If the reduced potential depends on a single scale $R$, i.e. $U(r)=-F(r / R) / R^{2}$, one gets universal scaling relations

$$
\frac{r_{0}}{R}=\bar{A}+\bar{B} \frac{R}{\alpha_{0}}+\bar{C} \frac{R^{2}}{\alpha_{0}^{2}},
$$

where $\bar{A}, \bar{B}$ and $\bar{C}$ are purely geometric numbers which depend solely on the functional form of the potential.

For the pure $\mathrm{VdW}$ case, $V=-C_{6} / r^{6}$, the effective range has been computed analytically [10, 11] and in harmony with the general structure Eq. (16) reads

$$
\frac{r_{0}}{R_{6}}=1.39473-1.33333 \frac{R_{6}}{\alpha_{0}}+0.637318 \frac{R_{6}^{2}}{\alpha_{0}^{2}},
$$

In Fig. 1 we confront the prediction for the effective range to the result of many potential calculations in $\mathrm{VdW}$ units. As can be vividly seen the agreement is rather impressive taking into account the simplicity of Eq. (17). Alternatively, and discarding the exceptional outliers for $\alpha_{0}$ 

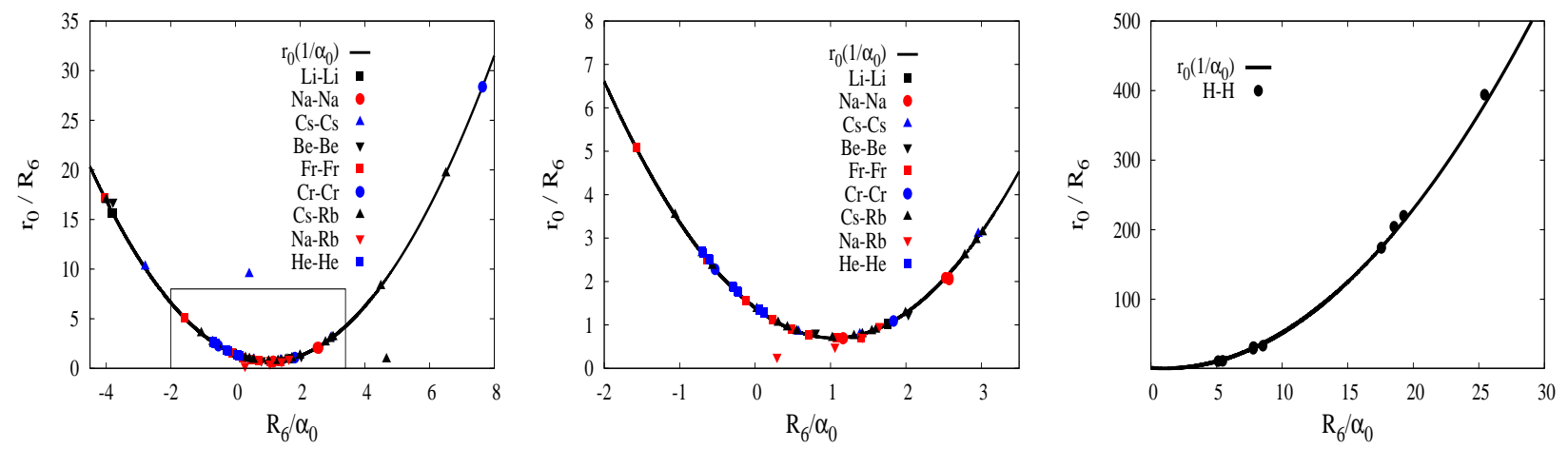

FIG. 1: The effective range $r_{0}$ vs the inverse scattering length $1 / \alpha_{0}$ in units of the VdW radius $R_{6}=\left(2 \mu C_{6} / \hbar^{2}\right)^{\frac{1}{4}}$ for different ranges. Points are potential calculations [24, 25, 26] (Li-Li,Na-Na), 27] (Cs-Cs), 28] (Na-Rb), 29] (Be-Be), 30] (Cs-Rb), 31] (Cr-Cr), 32] (Fr-Fr), 33, 34, 35, 36] (H-H), [36] (He-He). The line corresponds to Eq. (17) [10, 11].

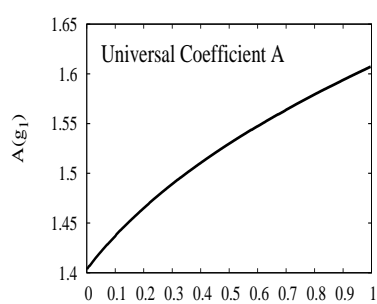

$g_{1}$

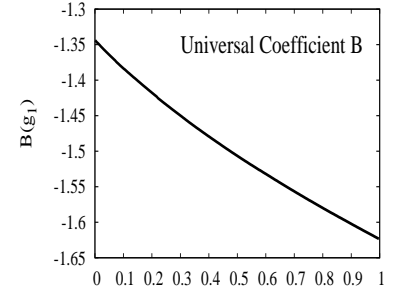

$\mathrm{g}_{1}$

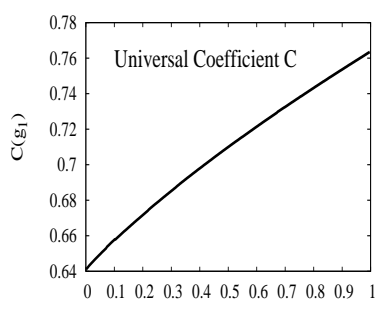

$\mathrm{g}_{1}$

FIG. 2: The effective range coefficients $A, B$ and $C$ (see Eq. (9)) as a function of the dimensionless coupling $g_{1}$ representing the $1 / r^{8}$ correction to the $\mathrm{VdW}$ potential $1 / r^{6}$.

and $r_{0}$, we perform a $\chi^{2}$ fit to the form Eq. (16) for the remaining 82 points and get $A=1.31, B=-1.57$ and $C=0.66$ in good agreement with Eq. (17). To our knowledge, Fig. 11 represents a completely unforeseen universal correlation supported by phenomenology.

We analyze the robustness of this agreement by showing in Fig. 2 the effect on the effective range coefficients, $A\left(g_{1}\right), B\left(g_{1}\right)$ and $C\left(g_{1}\right)$ due to adding a $1 / r^{8}$ term. From Table \we see that mostly $g_{1} \sim 10^{-2}$, in which case tiny changes are expected from Fig. 2. Actually, the smallness of the deviations suggests using perturbation theory. If we expand the full solutions of the $1 / r^{6}$ potential at small $k, u_{k}(r)=u_{0}(r)+k^{2} u_{2}(r)+\ldots$, the change in the effective range due to inclusion of a $\Delta U(r) \sim 1 / r^{8}$ potential keeping $\alpha_{0}$ fixed reads [4, 37]

$$
\Delta r_{0}=4 \int_{r_{c}}^{\infty} \Delta U(r) u_{0}(r) u_{2}(r) d r
$$

where $r_{c} \sim g_{1} R_{6} \ll R_{6}$. The leading contribution is determined by the short distance behaviour of $u_{0}(r)$, see Eq. (5), and we find $u_{2}(r) \sim\left(r / R_{6}\right)^{4} u_{0}(r)$ yielding $\Delta r_{0} \sim$ $g_{1} R_{6} \log g_{1}+\mathcal{O}\left(g_{1}\right)$. The logarithmic enhancement in $g_{1}$ can indeed be observed in Fig. 2 by the tiny curvature.

The $C_{6}$-dominance is compelling and we show in Fig. 3 the universal functions $\mathcal{A}(k), \mathcal{B}(k), \mathcal{C}(k)$ and $\mathcal{D}(k)$ which in conjunction with $\alpha_{0}$ allow to determine the phase-shift from Eq. (15). They scale with $R_{6}$ and are uniquely determined by the power law $-1 / r^{6}$ once and forever. We have found that these functions show little dependence on $g_{1}$ and $g_{2}$ at momenta as large as $k R_{6} \sim 10$, a rather unexpected result, hinting that the $\mathrm{VdW}$ universality and scaling extends much beyond the naive dimensional analysis estimate $k R_{6} \approx 1$ or the effective range expansion of Eq. (6) truncated with the first two terms. We note in passing that although such a truncation suggests a higher degree of universality, the $\mathrm{VdW}$ nature of the interaction prevents using $\left(\alpha_{0}, r_{0}\right)$ as fully independent variables in view of Eq. (17) and Fig. 1. It is interesting to notice that within a Nuclear Physics context characterized by short ranged Yukawa potentials stemming from meson exchange [38] the general Eq. (9) has been exploited as a means to check SU(4) Wigner symmetry in the NucleonNucleon interaction for the ${ }^{1} S_{0}$ and ${ }^{3} S_{1}$ channels with a pattern similar to Fig. 1].

We conclude by underlining that, when suitably displayed, the analytical approach to Van der Waals forces pursued in previous works [10, 11] acquires a quite universal character with indisputable phenomenological success; the leading $1 / r^{6}$ contribution suffices to accurately describe low energy atom-atom scattering with just two parameters in a wide energy range. We naturally expect new universality and scaling patterns to emerge 

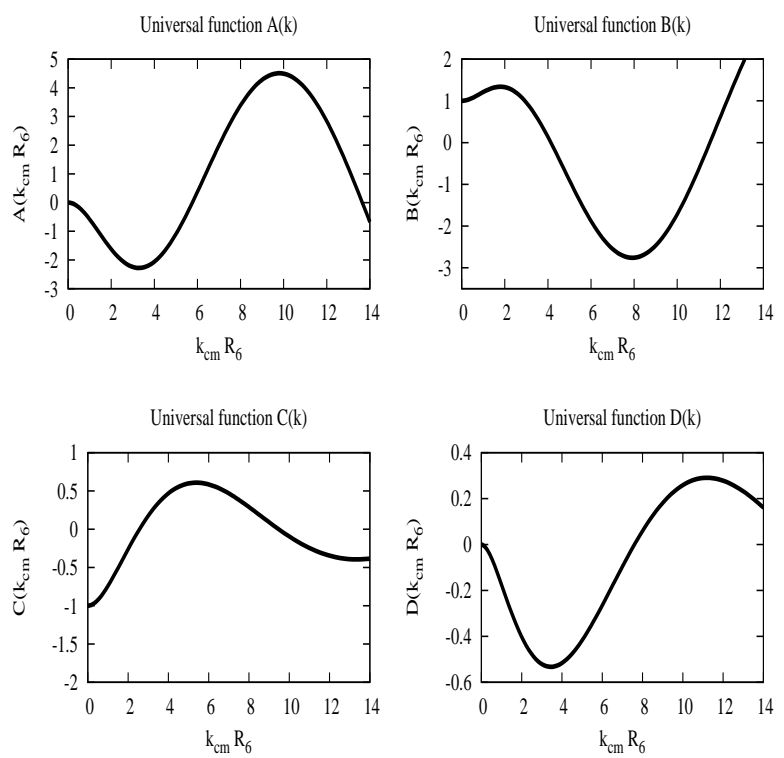

FIG. 3: The universal functions in units of $R_{6}$ defined by $2 \mu V(r)=-R_{6}^{4} / r^{6}$ which allow to determine the phase shift if the scattering length is also known, see Eq. (15).

from systems characterized by power law forces but less understood such as molecular interactions in the ultracold regime. From a broader perspective we stress that the lack of dependence of potential model calculations on short distance details, unveiled from our systematic comparison, is a feature traditionally built-in by the quantum-defect theory. Its natural counterpart of a smooth and controllable short distance cut-off dependence complies to the requirement of renormalizability within a pure quantum-mechanical framework.

We thank M. Pavón Valderrama and R. González Férez for discussions and M. Gacesa, P. Pellegrini and Z. Pavlović for comments. Work supported by the Ministerio de Ciencia y Tecnología, contract no. FIS200801143/FIS and Junta de Andalucía grant no.FQM225-05.

* Electronic address: alvarocalle@ugr.es

† Electronic address: earriola@ugr.es

[1] L. Boschke, van der Waals Systems (Springer, 1980).

[2] J. Weiner, Cold and Ultracold Collisions in Quantum Microscopic and Mesoscopic Systems (Cambridge University Press, 2004).

[3] R. Tarrach and M. Ericson, Nucl. Phys. A294, 417 (1978).

[4] M. Pavon Valderrama and E. R. Arriola, Phys. Rev. C74, 054001 (2006), nucl-th/0506047.

[5] G. Feinberg and J. Sucher, Phys. Rev. D20, 1717 (1979).

[6] H. Fujii and D. Kharzeev, Phys. Rev. D60, 114039 (1999), hep-ph/9903495.
[7] K. M. Case, Phys. Rev. 80, 797 (1950).

[8] W. Frank, D. J. Land, and R. M. Spector, Rev. Mod. Phys. 43, 36 (1971).

[9] M. Pavon Valderrama and E. R. Arriola, Annals Phys. 323, 1037 (2008), 0705.2952.

[10] B. Gao, Phys. Rev. A58, 1728 (1998).

[11] V. V. Flambaum, G. F. Gribakin, and C. Harabati, Phys. Rev. A 59, 1998 (1999).

[12] G. F. Gribakin and V. V. Flambaum, Phys. Rev. A 48, 546 (1993).

[13] B. Gao, Phys. Rev. A 80, 012702 (2009).

[14] S. V. Khristenko, A. I. Maslov, and V. P. Shevelko, Molecules and Their Spectroscopic Properties (Springer Series on Atoms and Plasmas. Vol. 21., 1998).

[15] F. C. Brooks, Physical Review 86, 92 (1952).

[16] J. Mitroy and M. W. J. Bromley, Phys. Rev. A 71, 032709 (2005), arXiv:physics/0411172.

[17] A. Derevianko, J. F. Babb, and A. Dalgarno, Phys. Rev. A 63, 052704 (2001), arXiv:physics/0102030.

[18] S. G. Porsev and A. Derevianko, J. Chem. Phys. 119, 844 (2003), arXiv:physics/0303048.

[19] M. Marinescu, D. Vrinceanu, and H. R. Sadeghpour, Phys. Rev. A 58, 4259 (1998).

[20] S. G. Porsev and A. Derevianko, Soviet Journal of Experimental and Theoretical Physics 102, 195 (2006).

[21] J. Werner, A. Griesmaier, S. Hensler, J. Stuhler, T. Pfau, A. Simoni, and E. Tiesinga, Physical Review Letters 94, 183201 (2005), arXiv:cond-mat/0412049.

[22] Z. Yan, J. F. Babb, A. Dalgarno, and G. W. F. Drake, Phys. Rev. A 54, 2824 (1996).

[23] B. R. Levy and J. B. Keller, Journal of Mathematical Physics 4, 54 (1963).

[24] R. Côté, E. J. Heller, and A. Dalgarno, Phys. Rev. A 53, 234 (1996).

[25] R. Côté, A. Dalgarno, and M. J. Jamieson, Phys. Rev. A 50, 399 (1994).

[26] R. Côté and A. Dalgarno, Phys. Rev. A 50, 4827 (1994).

[27] M. Marinescu, Phys. Rev. A 50, 3177 (1994).

[28] H. Ouerdane and M. J. Jamieson, European Physical Journal D 53, 27 (2009), 0802.1222.

[29] M. J. Jamieson, A. S-C Cheung, H. Ouerdane, G. Jeung, and N. Geum, Journal of Physics B Atomic Molecular Physics 40, 3497 (2007).

[30] M. J. Jamieson, H. Sarbazi-Azad, H. Ouerdane, G. Jeung, Y. S. Lee, and W. C. Lee, Journal of Physics B Atomic Molecular Physics 36, 1085 (2003).

[31] Z. Pavlović, B. O. Roos, R. Côté, and H. R. Sadeghpour, Phys. Rev. A 69, 030701 (2004), arXiv:physics/0309076.

[32] M. Kemal Öztürk and S. Özçelik, ArXiv Physics e-prints (2004), arXiv:physics/0406027.

[33] N. Koyama and J. C. Baird, Journal of the Physical Society of Japan 55, 801 (1986).

[34] M. J. Jamieson, A. Dalgarno, and J. N. Yukich, Phys. Rev. A 46, 6956 (1992).

[35] A. Sen, S. Chakraborty, and A. S. Ghosh, Europhysics Letters 76, 582 (2006).

[36] M. J. Jamieson, A. Dalgarno, and M. Kimura, Phys. Rev. A 51, 2626 (1995).

[37] M. P. Valderrama (2009), 0912.0699.

[38] A. Calle Cordon and E. Ruiz Arriola, Phys. Rev. C78, 054002 (2008), 0807.2918. 